\title{
Droplet Transportation on Vertical Parallel Electrodes Using Electrowetting and Interfacial Oscillation"
}

\author{
Hirofumi SHINTAKU**, Yukio TATARA** and Satoyuki KAWANO** \\ ${ }^{* *}$ Department of Mechanical Science and Bioengineering, Graduate School of Engineering Science, \\ Osaka University, \\ Machikaneyama-cho 1-3, Toyonaka, Osaka 560-8531, Japan \\ E-mail: shintaku@me.es.osaka-u.ac.jp
}

\begin{abstract}
In this study, we demonstrate the transportation of small liquid droplet on vertical parallel electrodes using electrowetting phenomena and the interfacial oscillation. The transportation of liquid droplets, which can be applied to droplet-based biochemical devices, is performed by applying $\mathrm{AC}$ voltage that ranges from 0 to $100 \mathrm{~V}_{\mathrm{pp}}$ and from 20 to $100 \mathrm{~Hz}$. The flow characteristics are observed by a high speed camera, and the effects of interfacial oscillation and the dynamic contact angle on the droplet motion are investigated in detail. The experimental results show that the vertical velocity of the droplet can be controlled by changing the amplitude and the frequency of the applied AC voltage. Furthermore, the dynamics of the droplet are estimated theoretically considering the interfacial tension due to the dynamic contact angle and the gravitational force. The predicted force and the displacement of the droplet reasonably agree with the experimental values obtained from the high speed observation of the droplet motion.
\end{abstract}

Key words: Electrowetting, Dynamics of Fluid Interface, Contact Angle, Droplet, MicroTAS

\section{Introduction}

The transportation of a small volume of liquid supported on a substrate is important technology in a variety of applications. For instance, it is fundamental for droplet-based micro total analysis systems ${ }^{(1)(2)}$. The electrostatic effect is useful for droplet transportation system from the viewpoint of fabrication, integration, and high controllability. Washizu demonstrated droplet transportation based on the principle of variable capacitance using an electrode array, as shown in Fig. 1 (a) ${ }^{(3)}$. In this method, different electric potentials are applied to a pair of electrodes, where one electrode is under the droplet and the other is in front of it. Then, the droplet moves toward the electrode in front of it due to Maxwell stress. By changing the pair of electrodes to apply the electric potential, the droplet is sequentially transported. Recently, electrowetting on dielectric (EWOD) is often used for droplet transportations ${ }^{(4) \sim(7)}$. EWOD is a phenomenon where the contact angle of liquids placed on insulated electrodes is decreased by the electrical force exerted on the edge region of the liquids. In other words, the electrical force makes the liquids more wettable on the surface of the insulated electrode. By inducing EWOD asymmetrically to a droplet, the droplet is transported to the more wettable region. This method requires both the integration of electrodes and the precise operation of electric potential for sequential transportation ${ }^{(4)(6)}$. On the other hand, Gunji et al. found a self-propelling phenomenon of water droplets on a pair of parallel electrodes ${ }^{(7)}$. Droplets are transported due to EWOD along parallel electrodes by applying AC voltage, as shown in Fig. 1 (b). Compared to other droplet 


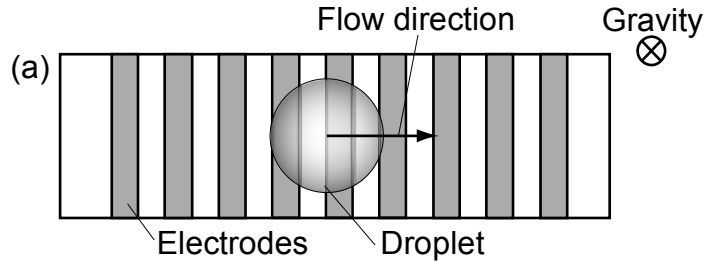

(b)

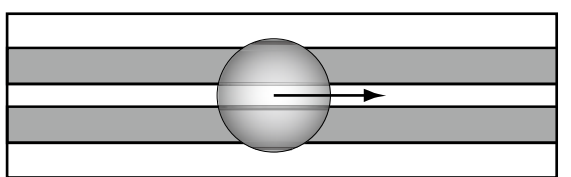

Fig. 1 Typical configurations of droplet transportation using an electrowetting device: railway sleepers (a) ${ }^{(3)}$ and railway $(b)^{(7)}$

transportation methods, the self-propelling system is easy to fabricate and operate. However, the transportation direction is highly dependent on the initial asymmetricity of the droplet shape. Thus, the flow direction of the droplet is relatively difficult to control. To develop a method for controlling the direction of self-propelling droplets, detailed investigation is required from the viewpoint of fluid dynamics.

In this study, droplet transportation on vertical parallel electrodes is explored to clarify the basic flow mechanism and to establish a controlling method of droplet transportation using the self-propelling phenomenon. One-directional transportation is realized inducing asymmetricity in the droplet shape using the gravitational force. Based on a high speed observation and a digital image processing ${ }^{(8)}$, the basic flow dynamics are clarified including the droplet transportation, the interfacial oscillation, and the contact angles. Furthermore, the dominant forces acting on the droplet transportation are theoretically predicted by considering the interfacial tension and the gravitational force. Comparing the predicted forces and the droplet motion with those of the experimental results, the basic mechanism of droplet transportation is discussed in detail.

\section{Nomenclature}

$C_{\mathrm{h} 1} \quad$ :electrostatic capacitance between electrodes through the insulation layer, $\mathrm{F}$

$c_{\mathrm{h} 2}$ :electrostatic capacitance per unit area between electrode and droplet, $\mathrm{F} / \mathrm{m}^{2}$

$C_{\mathrm{h} 2}$ :electrostatic capacitance between electrode and droplet, $\mathrm{F}$

$f \quad$ :frequency of applied voltage, $\mathrm{Hz}$

$F \quad$ :force acting on a droplet in $z$ direction, $\mathrm{N}$

$l \quad$ :width of droplet, $\mathrm{m}$

$m \quad$ :mass of droplet, $\mathrm{kg}$

$r \quad$ :radius of droplet, $\mathrm{m}$

$t \quad$ :time, $\mathrm{s}$

$U$ :velocity of droplet, $\mathrm{m} / \mathrm{s}$

$\bar{U} \quad$ :mean velocity of droplet, $\mathrm{m} / \mathrm{s}$

$V \quad$ :applied voltage, $\mathrm{V}$

$V_{\text {h2 }}$ :applied voltage acting on insulation layer, $\mathrm{V}$

$\bar{V} \quad$ :peak to peak amplitude of applied voltage, $\mathrm{V}_{\mathrm{pp}}$

$\bar{V}_{\mathrm{h} 2}$ :peak to peak amplitude of applied voltage on insulation layer, $\mathrm{V}_{\mathrm{pp}}$

$(x, y, z) \quad$ :Cartesian coordinates, $\mathrm{m}$

$\phi_{1}$ :phase delay of contact angle from applied voltage, deg.

$\gamma_{\mathrm{L}} \quad$ :surface tension of liquid, $\mathrm{N} / \mathrm{m}$

$\gamma_{\mathrm{LS}} \quad$ :interfacial tension between liquid and solid, $\mathrm{N} / \mathrm{m}$

$\gamma_{\text {LSO }}$ :interfacial tension between liquid and solid without applied voltage, $\mathrm{N} / \mathrm{m}$ 


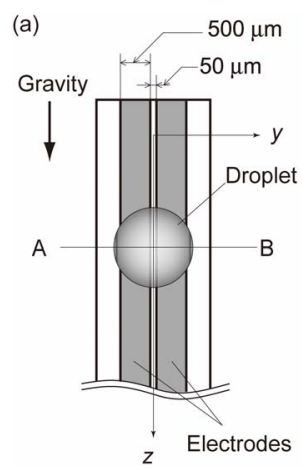

(b)

(c)

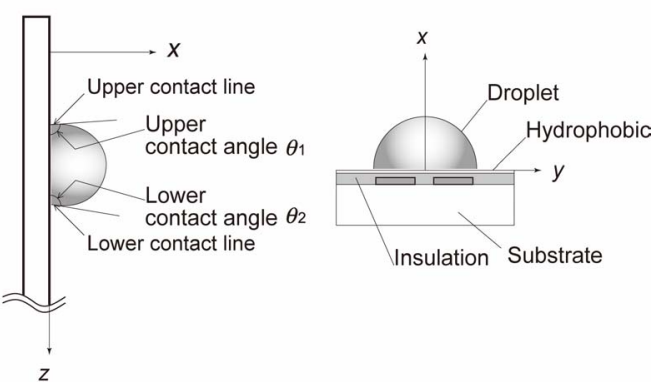

Fig. 2 Schematic of vertical parallel electrodes for droplet transportation in railway configuration: (a) front view, (b) side view, and cross sectional view at $\mathrm{AB}$

$\gamma_{\mathrm{S}} \quad$ :surface tension of solid, $\mathrm{N} / \mathrm{m}$

$\theta_{1} \quad$ :contact angle of liquid-solid interface, deg.

$\theta_{10} \quad$ :initial contact angle, deg.

$\rho \quad$ :density, $\mathrm{kg} / \mathrm{m}^{3}$

Subscripts

i : contact angles $\mathrm{i}=1$ or 2

1 :contact angle of upper half in droplet

2 :contact angle of lower half in droplet

\section{Mechanism and experimental methods of droplet transportation}

\subsection{Droplet transportation by EWOD}

Droplet transportation is achieved by EWOD in this study. When the droplet is set on the insulation layer with the applied voltage $V$, the interfacial tension $\gamma_{\mathrm{LS}}(V)$ can be described by the following equation: ${ }^{(4)}$

$$
\gamma_{\mathrm{LS}}(V)=\gamma_{\mathrm{LS} 0}-\frac{1}{2} c_{\mathrm{h} 2} V_{\mathrm{h} 2}^{2}
$$

where $c_{\mathrm{h} 2}=1.46 \times 10^{-4} \mathrm{~F} / \mathrm{m}^{2}, V_{\mathrm{h} 2}$ and $\gamma_{\mathrm{LS} 0}$ are the capacitance of the insulation layer per unit area, the electric potential applied to the layer and the interfacial tension at $V=0 \mathrm{~V}$, respectively. Electric potential $V_{\mathrm{h} 2}$ is part of total applied voltage $V$ and is estimated based on the equivalent electric circuit (see Appendix) to be half of $V \cdot \gamma_{\mathrm{LS}}(V)$ does not depend on the sign of $V$, since the change of $\gamma_{\mathrm{LS}}(V)$ is proportional to the square value of $V$. However, in practice, $\gamma_{\mathrm{LS}}(V)$ is affected by the sign at relatively high voltage, where this phenomenon is called as asymmetric electrowetting ${ }^{(9)}$. Since its effect is relatively small in this study, it is not discussed in detail. A schematic of the device including vertical parallel electrodes developed here is shown in Fig. 2. The device consists of a pair of parallel electrodes which are covered by an insulation layer. A stationary small droplet, which adheres to the surface of the electrodes, satisfies the static force balance between the interfacial tension and gravity without applying AC voltage. The deformation of droplet can be characterized by the upper contact angle and lower one, which are defined as $\theta_{1}$ and $\theta_{2}$, respectively, as shown in Fig. 2 (b). When the electrical voltage is applied to the electrodes, the droplet starts to deform. This deformation due to EWOD is caused by the flow dynamics of contact lines and of contact angles at the liquid-solid interface. Because of the dynamical deformation, the droplet starts to flow vertically downward as a result of the break of the static force balance. By applying AC voltage to the electrodes, the force balance is broken periodically. Thus, the droplet is observed to be continuously transported with interfacial oscillation from the macroscopic viewpoint. 


\subsection{Experimental setup}

The vertical parallel electrodes consist of a pair of planer electrodes that are rectangle-shaped of $500 \mu \mathrm{m}$ wide and $20 \mathrm{~mm}$ long. The gap between the two electrodes is set to be $50 \mu \mathrm{m}$. The fabrication process is briefly described as follows. Aluminum thin film with thickness of $100 \mathrm{~nm}$ is formed on a glass substrate by a sputtering technique. The film is processed to be the electrodes using the standard photolithography and etching methods. The electrodes are insulated by $500 \mathrm{~nm}$ thick polysilazane film (Polysilazane solution, AZ Electronic Materials). The surface is treated by Teflon (Teflon $\mathrm{AF}^{\circledR}$, DuPont) to make it hydrophobic. The contact angle of the water is measured to be $121 \mathrm{deg}$. on the Teflon surface of the electrodes when the electrodes are placed horizontally. The measured value is relatively larger than the typical one of $114 \mathrm{deg}$. for polytetrafluoroethylene. This may be owing to the surface roughness. However, the detailed mechanism is future work.

The processed substrate is fixed vertically for the present experiment. The experiment is conducted at the room temperature of $298 \mathrm{~K}$ controlled by air conditioner. Ultrapure water (Wako Pure Chemical Industries, Ltd.) is used for the water droplet. A small droplet with a volume of $2.0 \mu \mathrm{l}$ is initially at rest and is adhered at the center of the gap between the electrodes. The origin is set in the center of the initial position of the droplet. Cartesian coordinate is shown in Figs. 2 (a) (c). The flow dynamics of the droplet are recorded by a high speed camera (FASTCAM APX-RS, Photron). The velocities of the contact lines and the droplet are measured using sequential digital photographs by the image correlation method. In practice, the contact line's velocity is defined here by the velocity of the contact point at the liquid-solid interface in $z$ direction. The droplet velocity is measured using an interrogation window that includes the whole droplet. In this measurement, the displacement with sub-pixel precision is obtained by fitting the correlation value around the peak to the Gaussian function. Amplitude $\bar{V}$ and frequency $f$ of sinusoidal AC voltage are controlled from 0 to $100 \mathrm{~V}_{\mathrm{pp}}$ and from 20 to $100 \mathrm{~Hz}$, respectively. The time is counted from when the voltage starts to be applied.

The spatial precision of the digital images is $17.5 \mu \mathrm{m} /$ pixel. The temporal precision is controlled from 0.667 to $1.67 \mathrm{~ms}$ to obtain more than 30 images per an AC voltage cycle. The uncertainty in the measurement of the contact angle is approximately $0.5 \mathrm{deg}$.

\section{Results and discussion}

\subsection{Basic flow characteristics}

Figure 3 shows sequential photographs of droplet transportation at $50 \mathrm{~V}_{\mathrm{pp}}$ and $20 \mathrm{~Hz}$. Views in Figs. 3 (a) and (b) correspond to the observations shown in Figs. 2 (a) and (b), respectively. The droplet is at rest and adheres to the surface of the electrodes before AC voltage is applied. The equilibrium upper and lower contact angles are measured as $\theta_{1}=110$ deg. and $\theta_{2}=125 \mathrm{deg}$, respectively, at initial state defined as $t=0 \mathrm{~s}$. After applying AC voltage, the droplet oscillates and starts to flow vertically downwards due to EWOD. The displacement can be clearly confirmed by the photographs after $10(t=500 \mathrm{~ms})$ and 20 cycles $(t=1000 \mathrm{~ms})$. As confirmed by $\sin ^{2}(2 \pi f t)=(1-\cos (4 \pi f t)) / 2$ and Eq. $(1), \gamma_{\mathrm{LS}}(V)$ changes at the double frequency of the applied voltage. Thus, the frequency of the interfacial oscillation also doubles the value of the applied voltage. From Fig. 3 (a), the droplet seems spherical due to the small radius at $t=0 \mathrm{~s}$, but slightly extended in the vertical direction after the voltage is applied. If the droplet is misaligned to the center of the gap, the droplet is spontaneously moved to the center of the gap by applying the voltage, as shown in Fig. 4. This movement of the droplet may be caused by Maxwell stress ${ }^{(3)}$, which is induced by the permittivity difference between water and air.

The droplet position as a function of time is shown in Fig. 5. This result indicates that 
(a) Electrodes Droplet

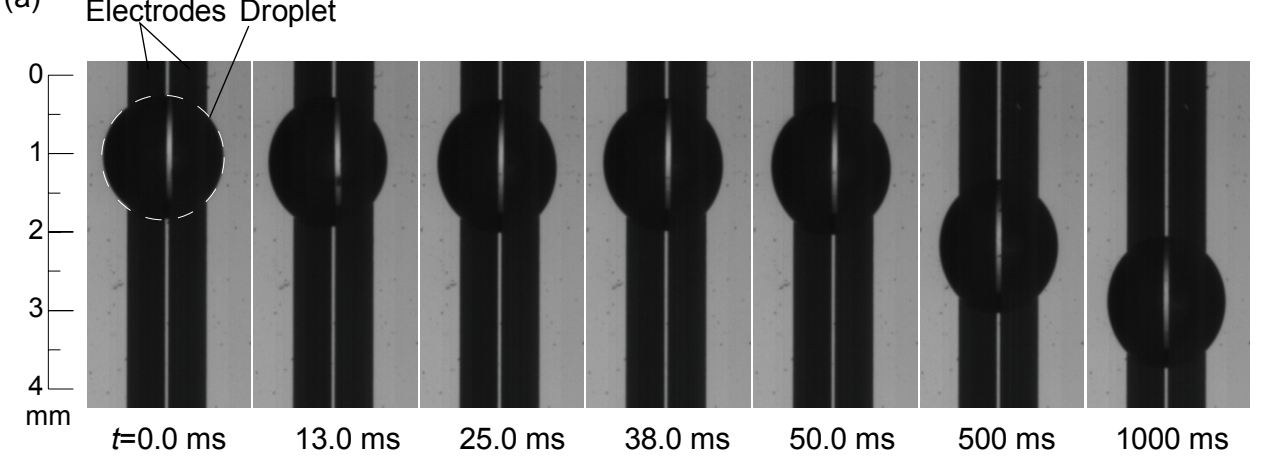

(b) Interface

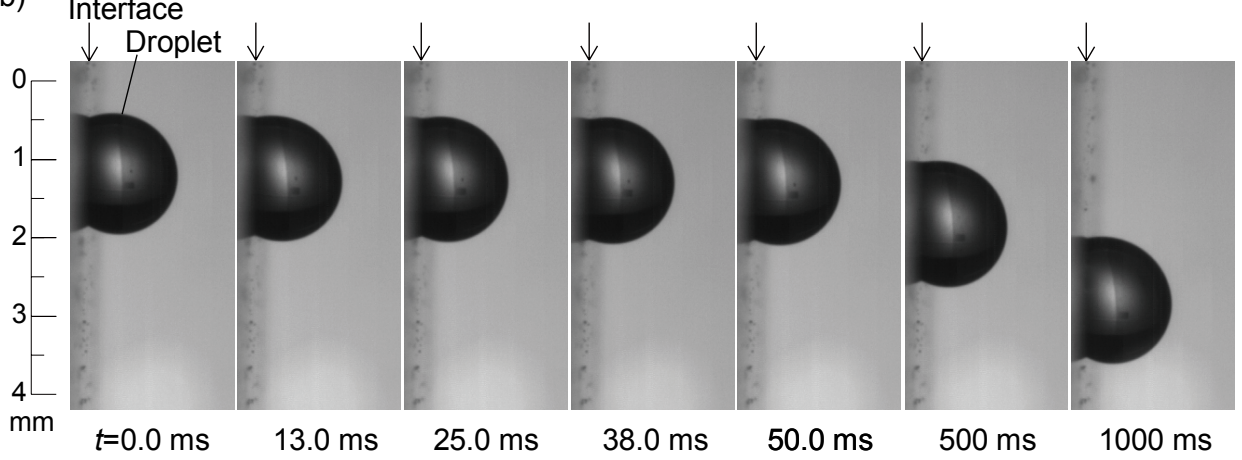

Fig. 3 Sequential photographs of droplet transportation observed from: (a) front and (b) side of vertical parallel electrodes at $\bar{V}=50 \mathrm{~V}_{\mathrm{pp}}$ and $f=20 \mathrm{~Hz}$

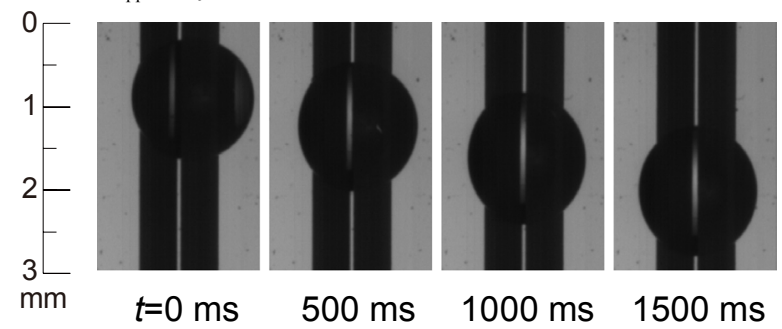

Fig. 4 Sequential photographs of misaligned droplet transportation at $\bar{V}=50 \mathrm{~V}_{\mathrm{pp}}$ and $f=50 \mathrm{~Hz}$

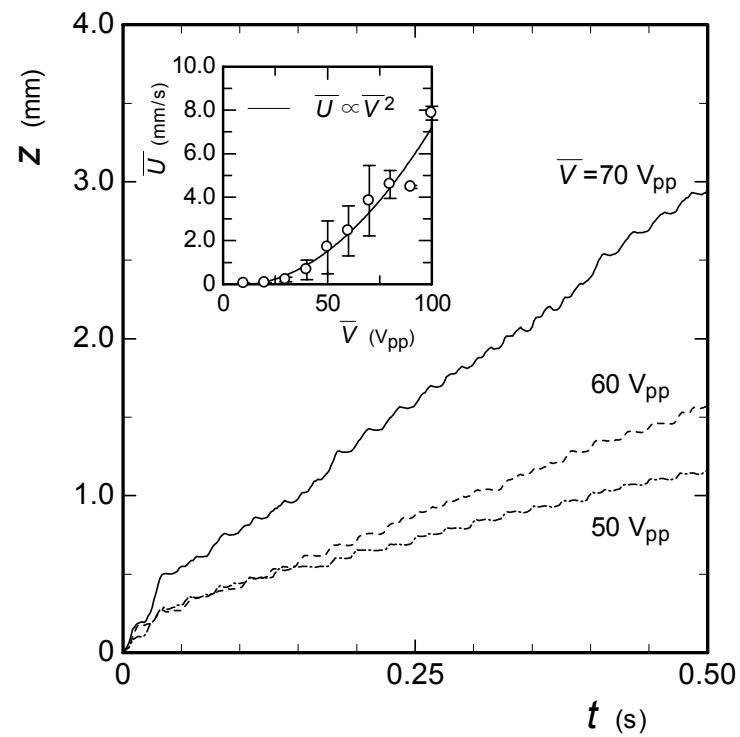

Fig. 5 Displacement $z$ and mean velocity $\bar{U}$ of droplet at various amplitude $\bar{V}$ of applied voltage at $f=20$ $\mathrm{Hz}$ 


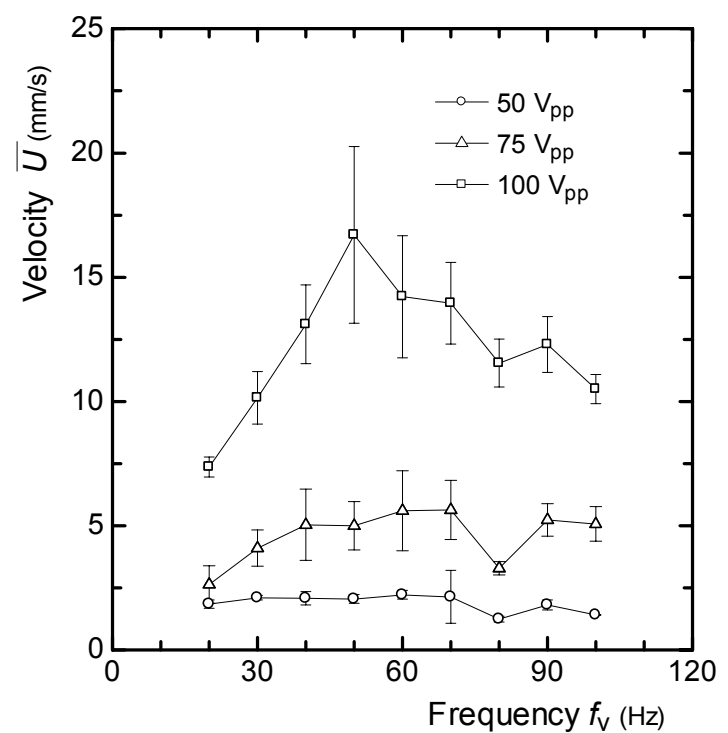

Fig. 6 Mean velocity $\bar{U}$ of droplet at various amplitudes $\bar{V}$ and frequency $f$ of applied voltage

the droplet is transported vertically downward with the vibration and seems to be transported at a constant rate per cycle, except for the early stage of the AC voltage. This suggests that transportation reaches a periodic steady state after the several cycles. The early stage, e.g., $t \cong 0 \sim 50 \mathrm{~ms}$ at $20 \mathrm{~Hz}$, is considered as a transition process from the stationary to the periodic steady state. Mean velocity $\bar{U}$ of the droplet at the periodic steady state increases in proportion to the square value of amplitude $\bar{V}$ at a certain frequency, as shown in the inset of Fig. 5. This may be related to the interfacial tension changes in proportion to $\bar{V}^{2}$. However, the detailed flow mechanism behind this relation remains an unsolved problem.

The relationship between frequency $f$ and mean velocity $\bar{U}$ is shown in Fig. $6 . \bar{U}$ looks independent on $f$ at $\bar{V}=50$ and $70 \mathrm{~V}_{\mathrm{pp}}$. On the contrary, $\bar{U}$ shows a clear peak around $f=45 \sim 55 \mathrm{~Hz}$ at $\bar{V}=100 \mathrm{~V}_{\mathrm{pp}}$. It may be that this peak is caused by the resonance of the interfacial oscillation. For the first step to theoretically investigate the phenomena, assuming that the droplet has a hemispherical shape with mobile contact lines and fixed contact angles of 90 deg., the oscillations of a droplet adhered on a substrate corresponds to that of a free droplet. Furthermore, since the motion of the contact lines in spherical coordinates seem to be almost the same at the upper and lower sides of the droplet due to the relatively strong EWOD effect rather than that of gravity. Thus, it may be said that the even mode of the interfacial oscillation is dominant in this system. To quantitatively explore the effect of $f$ on $\bar{U}$, the eigenfrequency of a spherical free droplet $^{(10)}$ is estimated with the assumptions of the stationary, inviscid, and small amplitude as

$$
f_{c}=(1 / 2 \pi) \sqrt{n(n-1)(n+2) \gamma_{L} / \rho r^{3}},
$$

where $r, n, \gamma_{\mathrm{L}}$, and $\rho$ are the radius, the mode number, the surface tension of the water, and the density, respectively. Based on the hemispherical droplet with a volume of $2.0 \mu$, the eigenfrequency is predicted to be $f_{\mathrm{c}}=124 \mathrm{~Hz}$ with values $r=9.85 \times 10^{-4} \mathrm{~m}, n=2, \gamma_{\mathrm{L}}=72 \times$ $10^{-3} \mathrm{~N} / \mathrm{m}$, and $\rho=998 \mathrm{~kg} / \mathrm{m}^{3}$. Mode number $n$ is determined to be $n=2$ based on experimental observation at $f=45 \sim 55 \mathrm{~Hz}$. The predicted frequency is roughly double the value of the $45 \sim 55 \mathrm{~Hz}$, indicating that the velocity increase is the result of the resonance due to the interfacial oscillation at $90 \sim 110 \mathrm{~Hz}$. Although the assumption of the hemispherical droplet leads to the overestimation of the droplet's radius which results in the 

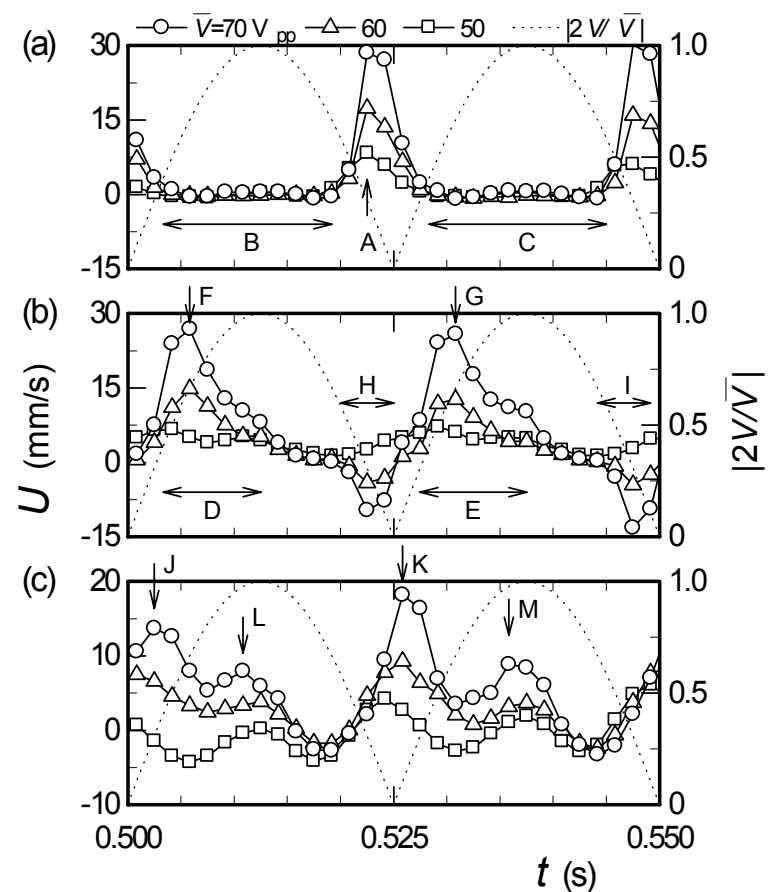

Fig. 7 Observed velocity $U$ in: (a) upper contact line, (b) lower contact line, and (c) droplet at $\bar{V}=50,60$, and $70 \mathrm{~V}_{\mathrm{pp}}$, respectively, and at $f=20 \mathrm{~Hz}$. Dotted line denotes absolute value $|2 V / \bar{V}|$ of scaled voltage

underestimation of the resonance frequency, the predicted resonance frequency of $124 \mathrm{~Hz}$ is higher than the measured one of $90 \sim 110 \mathrm{~Hz}$. The reason for the overestimation in the frequency is considered as the results of the overestimation in the surface tension, the finite amplitude of the oscillation, and the viscosity of the water in the experiment. The reason, why the effect of the resonance is clear at $100 \mathrm{~V}_{\mathrm{pp}}$ as shown in Fig. 6, is that the effect of the interfacial oscillation becomes stronger than that of liquid viscosity as $\bar{V}$ increases.

Transient velocity $U$ at the periodic steady state is shown in Fig. 7. Figures 7 (a), (b), and (c) are the velocities of the upper contact line, the lower contact line, and the droplet at $f=20 \mathrm{~Hz}$, respectively. Dotted lines denote absolute values $|2 V / \bar{V}|$ of the normalized voltage. It is found from Fig. 7 that the applied voltage has strong effect on the velocities change of the contact lines and the droplet. The peak positions of $U$ seem to be independent of the applied voltage. As shown in Fig. 7 (a), the upper contact line flows just before $|2 V / \bar{V}|$ approaches to 0 during the decrease, which is indicated as $\mathrm{A}$, but it is stationary during other periods of $\mathrm{B}$ and $\mathrm{C}$. Considering that the upper contact angle decreases during increase of $|2 V \overline{V V}|$, the lines should be moved to the outer direction from the droplet center. However, the upper contact line is stationary during $|2 V \bar{V}|$ increase in practice. This indicates that the upper contact line is difficult to flow to upward, since the droplet tends to flow downward due to the gravity. There are two cycles in the velocity change of the upper contact line per electric cycle. On the other hand, the lower contact line is fluctuated and flows when $|2 V \bar{V}|$ begins to increase from 0 , as shown $\mathrm{D}$ and $\mathrm{E}$ in Fig. 7 (b). After the velocity of the lower contact line shows local maximum value at $\mathrm{F}$ and $\mathrm{G}$, it gradually decreases until $|2 V / \bar{V}|$ starts to increase again. Just before $|2 V / \bar{V}|=0$, it is found that the velocity is the negative value at $\mathrm{H}$ and $\mathrm{I}$ at $\bar{V}=60$ and $70 \mathrm{~V}_{\mathrm{pp}}$. Comparing the absolute values of the velocity between the positive direction and the negative one, it is clear that the lower contact line tends to flow downwards. The velocity variation of the lower contact line seems rather complex than that of the upper contact line. This may be because the lower 


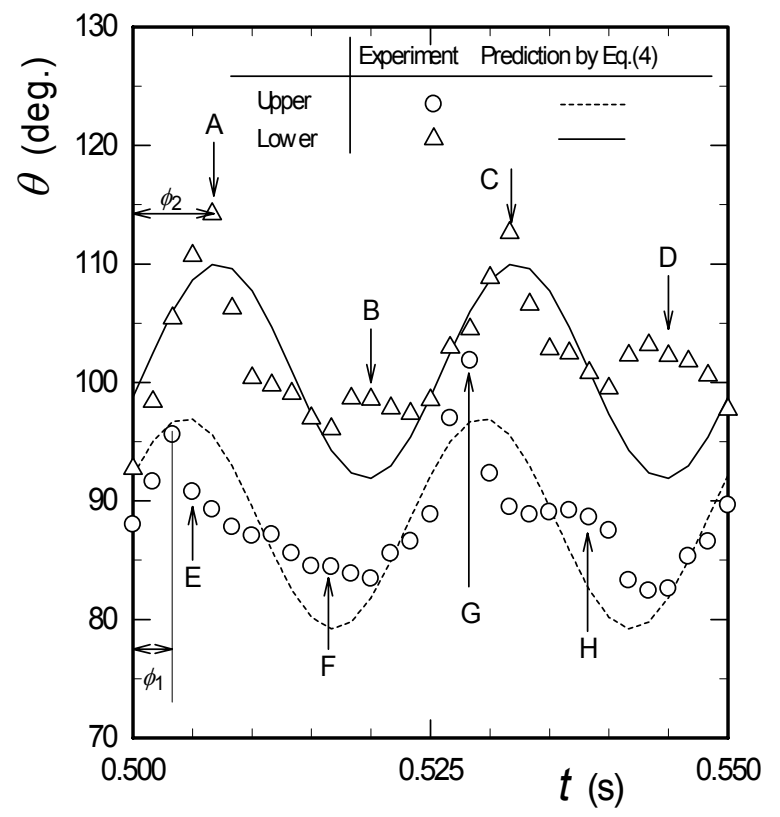

Fig. 8 Contact angle $\theta_{1}$ of droplet at upper and lower contact lines observed at $\bar{V}=70 \mathrm{~V}_{\mathrm{pp}}$ and $f=20 \mathrm{~Hz}$

contact line is not affected only by the change of the interfacial tension due to EWOD and the gravity but also by the dynamical motion of the droplet with the interfacial oscillation. Similar to the upper contact line, the velocity change of the lower contact line shows two cycles per an electric cycle. However, the upper contact line and the lower one do not flow simultaneously but alternately due to the effect of gravity, where they tend to flow in the gravity's direction. Comparing these contact lines, it can be said that the effect of the gravity is more dominant in the upper contact line, since the upper one shows only the positive velocity. In contrast to the contact lines, the droplet velocity roughly shows four peaks per an electric cycle as shown in Fig.7 (c), where the peaks consist of two higher ones of $\mathrm{J}$ and $\mathrm{K}$, and two lower ones of $\mathrm{L}$ and $\mathrm{M}$. The reason for the four peaks per an electric cycle must correspond to the result that the lower and upper contact lines flow alternately as described above. However, strictly speaking, the times of the peaks are not the same between the droplet and contact lines. The higher peak of $\mathrm{K}$ is found just after $|2 V \bar{V}|=0$, which is after the flow of upper contact line at A. The lower peaks of L and M are found shortly after the flow of lower contact line at $\mathrm{F}$ and $\mathrm{G}$, respectively. That is, the change of the interfacial tension due to EWOD firstly induces the contact lines' flow. Then, the contact lines' flow results the droplet's flow. These results suggest that the droplet is rather accelerating than flowing together with the contact lines. That is, when the contact lines are flowing dynamically, the static force balance is not maintained. Thus, the droplet is accelerated by the resultant force of the gravitational force and the dynamical interface tension.

\subsection{Contact angle}

The upper and lower contact angles at the periodic steady state are shown in Fig. 8. The solid and dotted lines are theoretical predictions that will be discussed later. The contact angles approximately change with the double frequency of the applied voltage, because the interfacial tension changes in response to the squared value of the applied voltage as shown in Eq.(1). Ideally, the contact angles should show the maximum values when $|2 V / \bar{V}|$ is zero, e.g., at $0.500,0.525$ and $0.550 \mathrm{~s}$, however, they clearly have the maximum values with phase delay $\phi_{1}$ from the voltage change. $\phi_{i}$ is determined experimentally as shown in Fig.8. 
This delay is more remarkable in the lower contact angle as $\phi_{1}<\phi_{2}$. All the contact angles at the periodic steady state are smaller than those at the initial condition. It is because the contact lines' flow is induced by the decrease of the interfacial tension $\gamma_{\mathrm{LS}}(V)$ in this system. Moreover, the maximum values are also smaller than those at initial condition. These results indicate the existence of the dynamical effect in the contact angles during the droplet motion.

To predict the change of the contact angles $\theta_{\mathrm{i}}$, a theoretical model at the periodic steady state is developed based on the experimental observations. In this development, we assume that the equation for the periodic steady state can be expressed by extending Young's equation that describes the static force balance of the interfacial tensions:

$$
\gamma_{\mathrm{LS}}(V)+\gamma_{\mathrm{L}} \cos \theta_{\mathrm{i}}(V)=\gamma_{\mathrm{S}},
$$

where $\gamma_{\mathrm{S}}$ and $\theta_{\mathrm{i}}(V)$ indicate the coefficient of the surface tension of the solid surface and the contact angle at $V$, respectively. Subscript $\mathrm{i}$ is 1 or 2 , where 1 and 2 correspond to the upper and the lower angles, respectively. Substituting Eq. (1) into Eq. (3), we obtain

$$
\theta_{\mathrm{i}}(V)=\arccos \left(\cos \theta_{\mathrm{i} 0}+\frac{c_{\mathrm{h} 2} \bar{V}_{\mathrm{h} 2}^{2} \sin ^{2}\left(360 f t+\phi_{\mathrm{i}}\right)}{8 \gamma_{\mathrm{L}}}\right),
$$

where $\bar{V}_{\mathrm{h} 2}$ and $\theta_{\mathrm{i} 0}$ are the peak to peak amplitude of $V_{\mathrm{h} 2}$ and the contact angle at $V=0$, respectively. The symbol of $\phi_{1}$ is used in Eq. (4) to describe the phase delay of the dynamical contact angles from the voltage change. The values of $\theta_{10}$ and $\phi_{1}$ are obtained by fitting Eq. (4) to the experimental data. The theoretical result by Eq. (4) is compared with the experimental data in Fig. 8. The upper and lower contact angles, which are predicted by Eq. (4), are shown by dotted and solid lines, respectively. The contact angles change with the double frequency of the applied voltage. The prediction of the lower contact angle deviates from the experimental data at $t=$ (A) 0.507 , (B) 0.520 , (C) 0.532 , and (D) $0.545 \mathrm{~s}$. That of the upper one deviates at $t=(\mathrm{E}) 0.505$, (F) 0.517, (G) 0.528, and (H) $0.538 \mathrm{~s}$. These deviations are found near the extrema of predicted curves and indicate the limitation of the theoretical model of Eq. (4), which does not include the dynamical effect except for the phase delay. Experimental results in Figs. 7 and 8 are compared in detail as follows. In the lower contact angle, their extrema at A, B, C, and D in Fig. 8 correspond to those in velocity of lower contact line around F, H, G and I in Fig.7 (b), respectively. Furthermore, in the upper contact angle, the extrema at E, F, G, and H in Fig.8 correspond to those around J, L, $\mathrm{K}$, and $\mathrm{M}$ in Fig.7 (c) where the droplet velocity shows the local maximum, respectively. The relationship between the droplet motion and the contact angle can be well clarified using the experimental results as described above, but the further discussion by the theoretical model including the dynamical interface tension should be made in future research.

\subsection{Dynamics of droplet transportation}

The theoretical model for droplet transportation must include the dynamical effect in the contact lines and angles in detail. However, this is quite difficult due to the lack of essential knowledge and the experimental data of this phenomenon. In this study, the basic principle of droplet transportation is discussed based on the experimental data. That is, the force is predicted using the contact angle model of Eq. (4) and is compared with the experimentally estimated force by the velocity change of the droplet. The dominant forces, which act on the droplet during transportation, are assumed to be the interfacial tension and gravity. Interfacial tension can be described using upper contact angle $\theta_{1}$ and lower one $\theta_{2}{ }^{(11)}$. Thus, the force balance can be written as

$$
F=m g-\gamma_{L} l\left(\cos \theta_{1}-\cos \theta_{2}\right)
$$




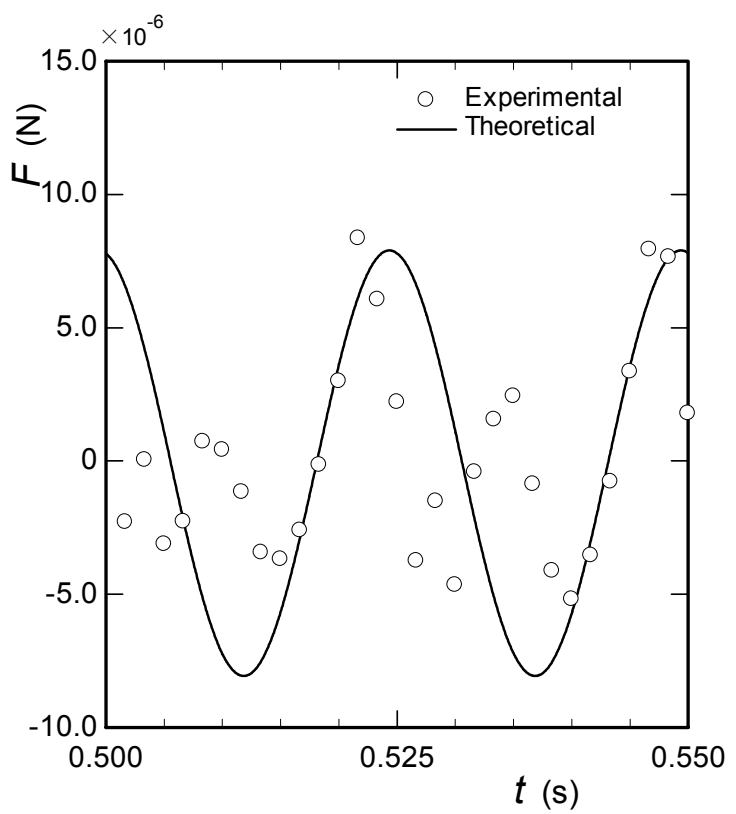

Fig. 9 Comparison of force $F$ exerted on droplet between experimental result and prediction from Eq. (5) at $\bar{V}=70 \mathrm{~V}_{\mathrm{pp}}$ and $f=20 \mathrm{~Hz}$

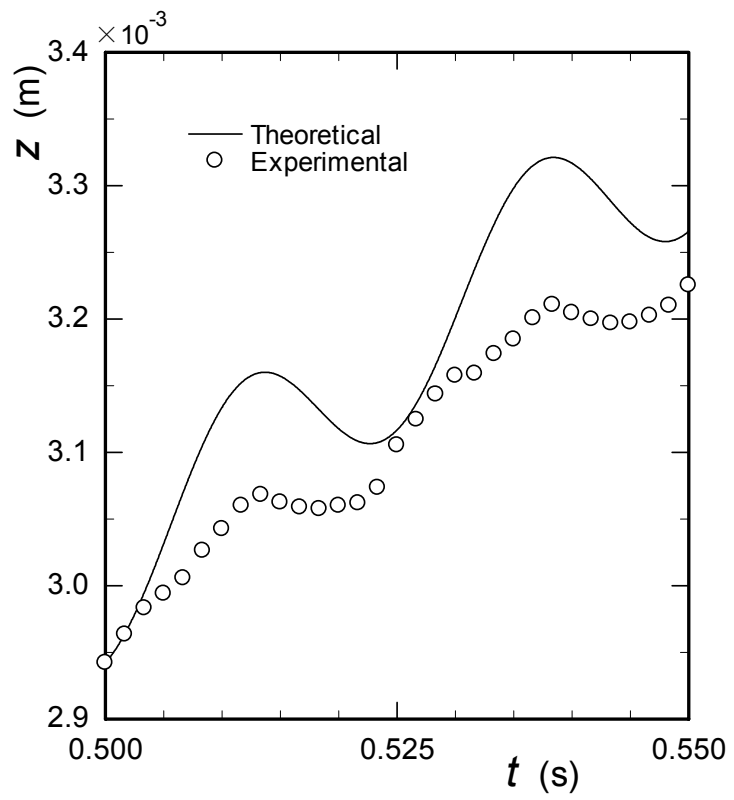

Fig. 10 Comparison of displacement $z$ between experimental result and prediction from Eq. (5) at $\bar{V}=70$ $\mathrm{V}_{\mathrm{pp}}$ and $f=20 \mathrm{~Hz}$

where $g, l$, and $m$ are gravitational acceleration of $9.81 \mathrm{~m} / \mathrm{s}^{2}$, the width of the droplet obtained to be $1.20 \mathrm{~mm}$ at the initial condition, and the mass of droplet of $2.00 \times 10^{-6} \mathrm{~kg}$, respectively. Figure 9 shows the comparison of the force $F$ between the prediction by Eq. (5) and the experimentally estimated values from the high-speed observations of the droplet motion. The maximum of the predicted force agrees well with that of the experiment at $t$ $=0.522$ and $0.547 \mathrm{~s}$. However, the prediction qualitatively deviates around $t=0.505 \sim 0.515$ $\mathrm{s}$ and $t=0.530 \sim 0.540 \mathrm{~s}$. The force from the experiment seems to show local maximum 
values four times per cycle, and the fact corresponds to the result in Fig. 7(c). On the other hand, the predicted value shows only twice per cycle. This qualitative difference may be caused by the prediction error in the dynamical contact angles as shown in Fig. 8, where it is our future work.

Figure 10 shows the comparison of the droplet transportation between the prediction by integrating the acceleration and the experimental data. For the initial conditions of theoretical predication, the position and the velocity at $t=0.500 \mathrm{~s}$ are used based on the experiment. The theoretical prediction reproduces well the qualitative dynamics of the droplet, i.e., the droplet flows downward viewed as a long period but can have negative velocity. Furthermore, it is found that the mean velocity is predicted to be locally fluctuated even though it seems constant in the experiment shown in Fig. 5. It is also found that there are the relatively large deviations from the experiment around the extrema of $z$ especially at $0.510 \sim 0.515 \mathrm{~s}$ and at $0.535 \sim 0.540$, which are almost the same periods shown in Fig. 9 . The deviation may be due to the energy dissipation by the viscous damping of the contact lines ${ }^{(12)}$, which is neglected in the present model. It is suggested that the viscous effect of the liquid should be included to improve the precision of prediction as well as the dynamics of contact lines of liquid-solid interface in future. However, the fundamental knowledge of the phenomena for theoretical understanding is herein presented as the first step.

\section{Conclusions}

In the present paper, the small droplet transportation on a pair of vertical parallel electrodes using the electrowetting and the interfacial oscillation was studied both experimentally and theoretically. The dynamics of the droplet motion, the contact lines, and the angles in EWOD were investigated using $2.0 \mu \mathrm{l}$ pure water droplets. The downward velocity of droplet could be successfully controlled by changing the amplitude $\bar{V}$ and the frequency $f$ of the applied voltage. The important results were obtained within the ranges of $\bar{V}=0 \sim 100 \mathrm{~V}_{\mathrm{pp}}$ and $f=20 \sim 100 \mathrm{~Hz}$ as follows.

(1) The mean velocity of droplet at the periodic steady state was considered to be constant at a certain condition of the applied voltage from the viewpoint of timescale above $O(0.1)$ s. The velocity could be controlled by changing the applied voltage, and was proportional to the square value of $\bar{V}$. Furthermore, a clear peak was found in the frequency dependence of the mean velocity at $\bar{V}=100 \mathrm{~V}_{\mathrm{pp}}$ and around $f=50 \mathrm{~Hz}$. This phenomenon was confirmed to be the resonance in the interfacial oscillation of the droplet because the resonant frequency and the oscillation mode were well predicted by theoretical analysis.

(2) The transient velocities of the upper and lower contact lines between the droplet and the solid surface at the periodic steady state showed the local maximum twice per cycle of the applied voltage. The contact lines flowed downwards due to the effect of the gravity not simultaneously but alternately. As the result of this alternate contact lines' motion, from the viewpoint of timescale in $O(0.01) \mathrm{s}$, the droplet showed the local maximum velocities four times per cycle of the applied voltage. Furthermore, the droplet seemed not to flow with contact lines, but to be accelerated by them in the small timescale, since the droplet showed the local maximum velocities shortly after the movement of each contact line.

(3) To predict the motion of the droplet, the simple theoretical model including the dynamical effect of phase delay between the applied voltage and the contact angles in EWOD was developed. The validity of the model was experimentally evaluated by comparing the force acting on the droplet. Through this evaluation, we found that the theoretical model could reproduce the qualitative characteristics of the droplet motion flowing not only downwards but also upwards with vibration in the small timescale of $O(0.01)$ s. However, the prediction of transportation showed over estimation from the 


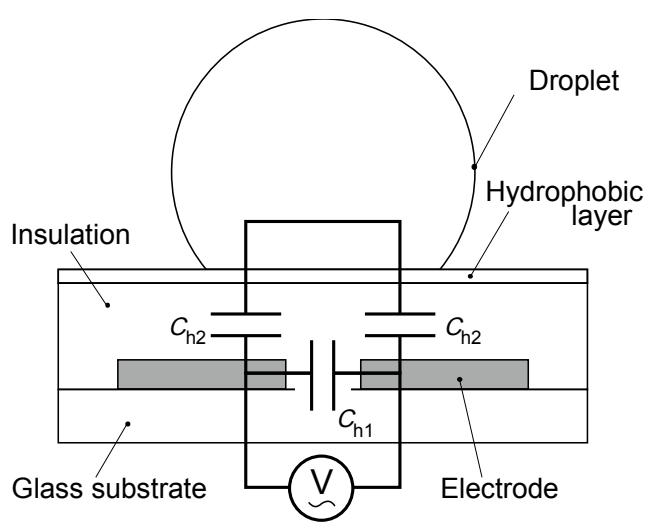

Fig. 11 Equivalent circuit for droplet and vertical parallel electrodes

experimental results when the droplet had a negative value of acceleration. These results indicated that the viscous damping of the contact lines and the dynamical interface tension of the droplet should be included in the theoretical model in future research.

\section{Appendix}

To estimate the voltage $V_{\mathrm{h} 2}$ on the insulation layer, the equivalent circuit as shown in Fig. 11 is used. The electrostatic capacitances shown as $C_{\mathrm{h} 1}$ and $C_{\mathrm{h} 2}$ in Fig. 11 are assumed to be constant during the droplet transportation, that is, they are independent of the droplet motion. $V_{\mathrm{h} 2}$ can be estimated as the voltage applied to $C_{\mathrm{h} 2}$, where $V_{\mathrm{h} 2}$ is half of $V$.

\section{References}

(1) Zeng, J. and Korsmeyer, T., Principles of Droplet Electrohydrodynamics for Lab-on-a-chip, Lab on a Chip, Vol. 4, Issue 4 (2004), pp. 265-277.

(2) Shintaku, H., Kuwabara, T., Kawano, S., Suzuki, T., Kanno, I., and Kotera, H., Micro Cell Encapsulation and Its Hydrogel-Beads Production Using Microfluidic Device, Microsystem Technologies, Vol. 13, No. 8-10 (2007), pp. 951-958.

(3) Washizu, M., Electrostatic Actuation of Liquid Droplets for Microreactor Applications, IEEE Transactions on Industry Applications, Vol. 34, No. 4 (1998), pp.732-737.

(4) Cho, S. K., Moon, H., and Kim, C.-J., Creating, Transporting, Cutting, and Merging Liquid Droplets by Electrowetting-Based Actuation for Digital Microfluidic Circuits, Journal of Microelectromechanical Systems, Vol. 12, No. 1 (2003), pp.70-80.

(5) Cooney, C. G., Chen, C-Y., Emerling, M. R., Nadim, A. and Sterling, J. D., Electrowetting Droplet Microfluidics on a Single Planar Surface, Microfluid Nanofluid, Vol. 2, No. 5 (2006), 435-446.

(6) Pollack, M. G., Shenderov, A. D., and Fair, R. B., Electrowetting-based Actuation of Droplets for Integrated Microfluidics, Lab on a Chip, Vol. 2, Issue 2(2002), pp. 96-101.

(7) Gunji, M. and Washizu, M., Self-propulsion of a Water Droplet in an Electric Field, Journal of Physics D: Applied Physics, Vol. 38, No. 14 (2005), pp. 2417-2423.

(8) Shintaku, H. Imamura, S. and Kawano, S., Microbubble Formations in MEMS-Fabricated Rectangular Channels: A High-Speed Observation, Experimental Thermal and Fluid Science, Vol.32, Issue 5(2008), pp.1132-1140.

(9) Fan, S., Yang, H., Wang, T., and Hsu, W., Asymmetric electrowetting-moving droplets by a square wave, Lab on a Chip, Vol.7, Issue 10 (2007), pp. 1330-1335.

(10) Kawano, S., Hashimoto, H., Ihara, A., and Azima, T., Small-Amplitude Oscillations of Encapsulated Liquid Drop Interfaces, JSME International Journal. Ser. B, Fluids and thermal engineering, Vol. 40, No. 1(1997), pp. 33-41.

(11) Dussan V., E. B. and Chow, R.T. P., On the Ability of Drops or Bubbles to Stick to Non-horizontal Surfaces of Solids, Journal of Fluid Mechanics, Vol. 137 (1983), pp.1-29.

(12) Lyubimov, D.V., Lyubimova, T. P., and Shklyaev, S.V., Behavior of a Drop on an Oscillating Solid Plate, Physics of Fluids, Vol. 18(2006), pp. (012101-1)- (012101-11). 